\title{
Decode-and-Forward Differential Modulation Scheme with Threshold-Based Decision Combining
}

\author{
Thanongsak Himsoon, W. Pam Siriwongpairat, Weifeng Su ${ }^{\ddagger}$, and K. J. Ray Liu \\ Department of Electrical and Computer Engineering, University of Maryland, College Park, MD 20742 \\ $\ddagger$ Department of Electrical Engineering, State University of New York (SUNY) at Buffalo, Buffalo, NY 14260
}

\begin{abstract}
This paper proposes a threshold-based differential decode-and-forward cooperative scheme that efficiently exploits the cooperative relay channels via the use of a pre-determined decision threshold. In the proposed scheme, the source information is forwarded by the relay only if it is correctly decoded. The properlydesigned threshold enables the destination to decide whether the received signal from the relay contains information such that the received signals from the source and the relay can be efficiently combined and jointly decoded. The bit error rate (BER) analysis of the proposed scheme is analyzed in case of differential M-ary phase shift keying signals. A tight BER approximation is established, and BER upper bound and lower bound are determined. Based on the tight BER approximation, joint optimum decision threshold and power allocation is numerically evaluated. Both analytical and simulation results reveal that the decision threshold and the power allocation depend on channel link qualities. Interestingly, when the link quality between the relay and the destination is very good, the effect of the threshold dominates the effect of the power allocation at high signal-to-noise ratio. Extensive simulation results are provided to validate the merit of the proposed scheme and confirm the theoretical analysis.
\end{abstract}

\section{INTRODUCTION}

In conventional communication systems, differential detection with diversity combining is considered as an attractive alternative to coherent detection that provides a good tradeoff between receiver complexity and performance [1]. No need of channel estimation has attracted many researchers to deploy differential detection in multiple-input multiple-output (MIMO) systems [2]. However, the deployment of multi-antenna terminals may be difficult in some applications since the mobile terminals are practically small. Recently, an idea of resource sharing among users has been introduced as cooperative communication paradigm [3][4] that explores inherent spatial diversity through relay channels. Different cooperation protocols are proposed based on relay processing such as amplify-and-forward (AF), decode-and-forward (DF) [3]-[4], and coded cooperation protocol [5].

Most of the existing works in [4]-[8] and references threrin assume that the destination has perfect knowledge of channel state information (CSI). While the CSI is likely to be acquired in slow fading by the use of pilot symbols, it may not be possible in fast fading environment. In addition, it is questionable on how the destination obtain the source-relay channel perfectly without noise amplification. Moreover, the computational overhead for channel estimation increases in proportional to the number of transmit antennas and relays. To overcome such problems and reduce receiver complexity, a specific two-hop relay system using differential modulation has been investigated in [6]. In [9], a framework of noncoherent cooperative diversity has been proposed for the DF protocol employing frequency shift keying signals. However, the framework does not fit to the general differential M-ary phase shift keying (DMPSK). A specific two-user differential cooperation scheme was proposed in [10]. However, the scheme relies on synchronization among users, and provides limited transmission rate.

In this paper, we propose a threshold-based differential cooperative scheme employing the DF protocol. The scheme efficiently exploits the inherent spatial diversity in the relay channels through the use of a pre-determine decision threshold. The destination makes judgement based on the properly-designed threshold whether to combine signal from the relay with the signal from the source before being jointly decoded. We analyze the bit error rate (BER) performance of the proposed scheme employing DMPSK signals. A tight approximate BER formulation together with its upper bound and lower bound are provided. Optimum decision threshold and power allocation are jointly designed based on the tight BER approximation. Simulation results are shown to validate our proposed schemes and support our analytical analysis.

\section{The Proposed Scheme}

Consider a two-user cooperation system as shown in Figure 1 in which signal transmissions involve two transmission phases. In both phases, we assume that all users transmit their signals through orthogonal channels by the use of existing schemes such as TDMA, FDMA, or CDMA.

In Phase I, the source differentially encodes the information symbol as

$$
x^{\tau}=v_{m} x^{\tau-1},
$$

where $\tau$ is the time index, $x^{\tau}$ is the differentially encoded symbol to be transmitted at time $\tau$, and $v_{m}=e^{j \phi_{m}}$ is the set of information symbols to be transmitted by the source. For DMPSK signals, $\phi_{m}$ is specified as $\phi_{m}=2 \pi m / M$ for $m=0,1, \ldots, M-1$. Based on the transmitted symbol $x^{\tau}$, the received signals at the destination and the relay can be expressed as

$$
\begin{aligned}
& y_{s, d}^{\tau}=\sqrt{P_{1}} h_{s, d}^{\tau} x^{\tau}+w_{s, d}^{\tau}, \\
& y_{s, r}^{\tau}=\sqrt{P_{1}} h_{s, r}^{\tau} x^{\tau}+w_{s, r}^{\tau},
\end{aligned}
$$

where $h_{s, d}^{\tau}$ and $h_{s, r}^{\tau}$ are channel coefficients at the sourcedestination link and the source-relay link, respectively, and $w_{s, d}^{\tau}$ and $w_{s, r}^{\tau}$ are additive noise. Both channel coefficients $h_{s, d}^{\tau}$ and $h_{s, r}^{\tau}$ are modeled as zero-mean complex Gaussian random variables with variances $\sigma_{s, d}^{2}$ and $\sigma_{s, r}^{2}$, i.e., $\mathcal{C N}\left(0, \sigma_{s, d}^{2}\right)$ and $\mathcal{C N}\left(0, \sigma_{s, r}^{2}\right)$, respectively. Each of the noise terms is modeled as $\mathcal{C N}\left(0, \mathcal{N}_{0}\right)$ where $\mathcal{N}_{0}$ is the noise power spectral density.

In Phase II, the relay differentially decodes the transmitted symbol from the source by using the decision rule: [11]

$$
\hat{m}=\arg \max _{m=0,1, \ldots, M-1} \operatorname{Re}\left\{\left(v_{m} y_{s, r}^{\tau-1}\right)^{*} y_{s, r}^{\tau}\right\} .
$$

In the proposed scheme, we assume that the relay can judge 


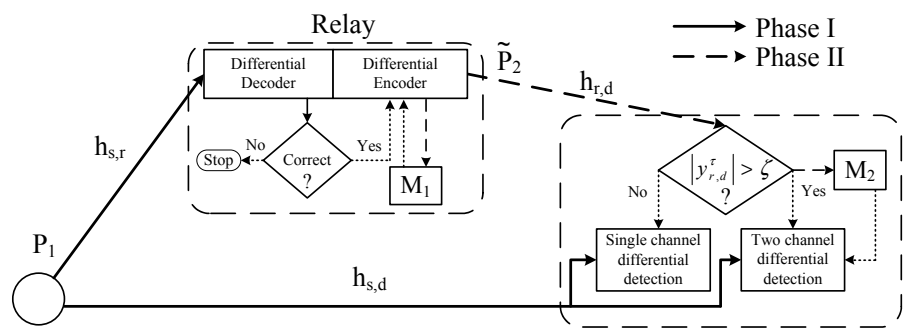

Source

Destination

Fig. 1: System descriptions of the proposed differential scheme.

whether the decoded information is correct or not ${ }^{1}$. If the relay incorrectly decodes the received signal, such incorrectly decoded symbol is discarded and the transmitted power at the relay is $\tilde{P}_{2}=0$. Otherwise, the relay differentially re-encodes the correctly decoded information symbol as $\tilde{x}^{\tau}=v_{m} \tilde{x}^{\tau-k}$, and $\tilde{x}^{\tau}$ is forwarded to the destination with transmitted power $\tilde{P}_{2}=P_{2}$. In addition, the transmitted symbol $\tilde{x}^{\tau}$ is stored in the memory $M_{1}$ to be used for subsequent differential encoding. Note that the previously transmitted symbol $\tilde{x}^{\tau-k}$ can be any time before time $\tau$ depending on the decoding result at the relay. Accordingly, the received signal at the destination from the relay in Phase II can be expressed as

$y_{r, d}^{\tau}= \begin{cases}\sqrt{P_{2}} h_{r, d}^{\tau} \tilde{x}^{\tau}+w_{r, d}^{\tau}, & \text { if relay correctly decodes }\left(\tilde{P}_{2}=P_{2}\right) ; \\ w_{r, d}^{\tau}, & \text { Otherwise }\left(\tilde{P}_{2}=0\right),\end{cases}$ where $h_{r, d}^{\tau} \sim \mathcal{C} \mathcal{N}\left(0, \sigma_{r, d}^{2}\right)$ is the channel coefficient at the relaydestination link and $w_{r, d}^{\tau} \sim \mathcal{C N}\left(0, \mathcal{N}_{0}\right)$ is an additive noise. Without knowledge of the CSI, the destination is unable to know whether the received signal from the relay contains the information or not. In order for the destination to judge whether to combine the signals from the source-destination and relaydestination links, we propose to use a threshold $\zeta$ to make decision based on the value of $\left|y_{r, d}^{\tau}\right|$ (see Figure 1). Therefore, the combined signal at the destination can be written as

$$
y= \begin{cases}\left(y_{s, d}^{\tau-1}\right)^{*} y_{s, d}^{\tau} & \text { if }\left|y_{r, d}^{\tau}\right| \leq \zeta \\ a_{1}\left(y_{s, d}^{\tau-1}\right)^{*} y_{s, d}^{\tau}+a_{2}\left(y_{r, d}^{\tau-l}\right)^{*} y_{r, d}^{\tau} & \text { if }\left|y_{r, d}^{\tau}\right|>\zeta .\end{cases}
$$

where $a_{1}$ and $a_{2}$ are combining weights, and $\tau-l(l \geq 1)$ represents the time index of the latest signal in memory $M_{2}$, i.e., $y_{r, d}^{\tau-l}$ is the most recent received signal from the relay whose amplitude is larger than the threshold. From (5), we can see that if $\left|y_{r, d}^{\tau}\right| \leq \zeta$, the destination estimates the transmitted symbol based only on the received signal from the direct link. However, when $\left|y_{r, d}^{\tau}\right|>\zeta$, the received signal from the source and that from the relay are combined for jointly decoding. Based on the combined signal in (5), the destination jointly differentially decodes by

$$
\hat{m}=\arg \max _{m=0,1, \ldots, M-1} \operatorname{Re}\left\{v_{m}^{*} y\right\} .
$$

Note that using different combining weights $\left(a_{1}\right.$ and $\left.a_{2}\right)$ affect the system performance. In this paper, we use $a_{1}=a_{2}=1 /\left(2 \mathcal{N}_{0}\right)$, and we will show later that these combining weights maximize the signal-to-noise ratio (SNR) of the combiner output.

\section{BER ANALYSIS}

We first classify possible scenarios that result in different instantaneous SNRs, then the probability of occurrence of each scenario is determined, and finally average BER is derived.

${ }^{1}$ Practically, this can be done at the relay by applying a simple SNR threshold on the received data. Although, error propagation may occurs, but for practical operating SNR ranges, the event of error propagation can be assumed negligible.

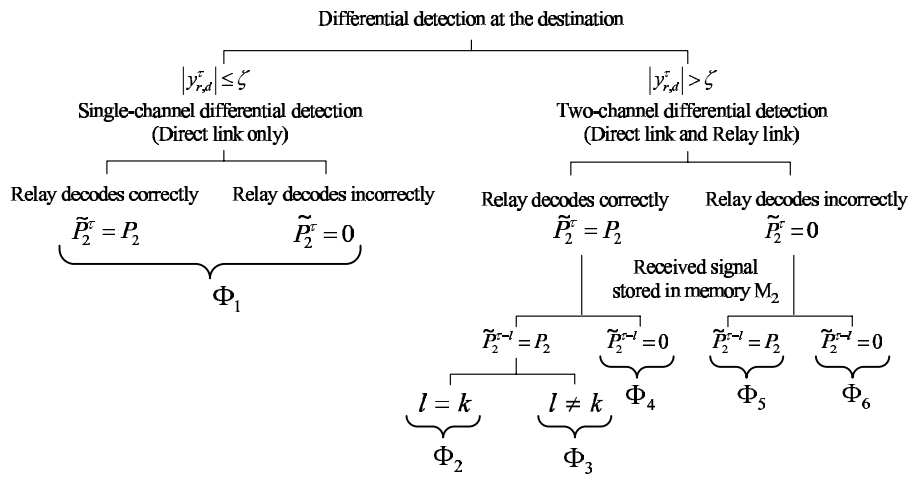

Fig. 2: Six possible scenarios based on the currently received signal and the signal in memory $M_{2}$.

\section{A. Classification of Different Scenarios}

Depending on whether the relay correctly decodes or not, and the level of $\left|y_{r, d}^{\tau}\right|$ comparing to the threshold $\zeta$, we can classify six possible scenarios that result in different SNR's at the combiner output as summarized in Figure 2. We denote each scenario as $\Phi_{i}, i=1,2, \ldots, 6$. Due to space limitation, we show the results of these six scenarios here:

$$
\begin{aligned}
& \Phi_{1} \triangleq\left\{\left|y_{r, d}^{\tau}\right| \leq \zeta\right\}, \\
& \Phi_{2} \triangleq\left\{\left|y_{r, d}^{\tau}\right|>\zeta, \tilde{P}_{2}^{\tau}=P_{2}, \tilde{P}_{2}^{\tau-l}=P_{2}, l=k\right\}, \\
& \Phi_{3} \triangleq\left\{\left|y_{r, d}^{\tau}\right|>\zeta, \tilde{P}_{2}^{\tau}=P_{2}, \tilde{P}_{2}^{\tau-l}=P_{2}, l \neq k\right\}, \\
& \Phi_{4} \triangleq\left\{\left|y_{r, d}^{\tau}\right|>\zeta, \tilde{P}_{2}^{\tau}=P_{2}, \tilde{P}_{2}^{\tau-l}=0\right\}, \\
& \Phi_{5} \triangleq\left\{\left|y_{r, d}^{\tau}\right|>\zeta, \tilde{P}_{2}^{\tau}=0, \tilde{P}_{2}^{\tau-l}=P_{2}\right\}, \\
& \Phi_{6} \triangleq\left\{\left|y_{r, d}^{\tau}\right|>\zeta, \tilde{P}_{2}^{\tau}=0, \tilde{P}_{2}^{\tau-l}=0\right\} .
\end{aligned}
$$

As an example, $\Phi_{2}$ indicates joint events that include $\left|y_{r, d}^{\tau}\right|>\zeta$, the relay correctly decodes at time $\tau$ and $\tau-1$, and the symbols at time $k$ and $l$ are the same. Other scenarios can be interpreted in a similar way.

\section{B. Probability of Occurrence}

With DMPSK signals, the chance of incorrect decoding at the relay, i.e. $\tilde{P}_{2}^{\tau}=0$, can be obtained from the conditional symbol error rate as [12]

$$
P_{r}^{h}\left(\tilde{P}_{2}^{\tau}=0\right)=\Psi\left(\gamma_{s, r}^{\tau}\right) \triangleq \frac{1}{\pi} \int_{0}^{(M-1) \pi / M} \exp \left[-g(\theta) \gamma_{s, r}^{\tau}\right] d \theta
$$

where $\gamma_{s, r}^{\tau}=P_{1}\left|h_{s, r}^{\tau}\right|^{2} / \mathcal{N}_{0}$ is an instantaneous SNR per symbol at the relay, and $g(\theta)=\frac{\sin ^{2}(\pi / M)}{1+\cos (\pi / M) \cos (\theta)}$. Therefore, the chance of correct decoding at the relay is $P_{r}^{h}\left(\tilde{P}_{2}^{\tau}=P_{2}\right)=1-\Psi\left(\gamma_{s, r}^{\tau}\right)$. Hence, from (7), the chance that $\Phi_{1}$ occurs can be written as

$$
\begin{aligned}
P_{r}^{h}\left(\Phi_{1}\right)= & \left(1-\exp \left(-\zeta^{2} / \mathcal{N}_{0}\right)\right) \Psi\left(\gamma_{s, r}^{\tau}\right) \\
& +\left(1-\mathcal{M}\left(P_{2}\left|h_{r, d}^{\tau}\right|^{2}, \zeta\right)\right)\left[1-\Psi\left(\gamma_{s, r}^{\tau}\right)\right],
\end{aligned}
$$

where we substitute $P_{r}^{h}\left(\left|y_{r, d}^{\tau}\right| \leq \zeta \mid \tilde{P}_{2}^{\tau}=0\right)=1-$ $\exp \left(-\zeta^{2} / \mathcal{N}_{0}\right), \quad P_{r}^{h}\left(\left|y_{r, d}^{\tau}\right| \leq \zeta \mid \tilde{P}_{2}^{\tau}=P_{2}\right)=1-$ $\mathcal{M}\left(P_{2}\left|h_{r, d}^{\tau}\right|^{2}, \zeta\right)$, and

$$
\mathcal{M}\left(P_{2}\left|h_{r, d}^{\tau}\right|^{2}, \zeta\right) \triangleq Q_{1}\left(\sqrt{P_{2}\left|h_{r, d}^{\tau}\right|^{2} /\left(\mathcal{N}_{0} / 2\right)}, \zeta /\left(\sqrt{\mathcal{N}_{0} / 2}\right)\right),
$$

in which $Q_{1}(\alpha, \beta)$ denotes the Marcum Q-function [11].

The chance that each of the scenarios $\Phi_{2}$ to $\Phi_{6}$ happens will be conditioned on the event that $\left|y_{r, d}^{\tau-l}\right|>\zeta$ because the destination has prior knowledge that $\left|y_{r, d}^{\tau-l}\right|>\zeta$ is stored in the memory $M_{2}$. From (8), the chance that $\Phi_{2}$ occurs is given by 


$$
\begin{aligned}
P_{r}^{h}\left(\Phi_{2}\right)= & P_{r}^{h}\left(\left|y_{r, d}^{\tau}\right|>\zeta, \tilde{P}_{2}^{\tau}=P_{2}\right) \\
& \times P_{r}^{h}\left(\tilde{P}_{2}^{\tau-l}=P_{2}, l=k|| y_{r, d}^{\tau-l} \mid>\zeta\right) \\
\approx & \frac{\mathcal{M}^{2}\left(P_{2}\left|h_{r, d}^{\tau}\right|^{2}, \zeta\right)\left(1-\Psi\left(\gamma_{s, r}^{\tau}\right)\right)^{2}}{1-\left(1-e^{-\zeta^{2} / \mathcal{N}_{0}}\right) \Psi\left(\gamma_{s, r}^{\tau}\right)},
\end{aligned}
$$

in which the first equality results from independent of events at time $\tau-l$ and time $\tau$. The approximation in (16) is obtained by rewriting the first term in the equality of (16) as $P_{r}^{h}\left(\left|y_{r, d}^{\tau}\right|>\right.$ $\left.\zeta, \tilde{P}_{2}^{\tau}=P_{2}\right)=\mathcal{M}\left(P_{2}\left|h_{r, d}^{\tau}\right|^{2}, \zeta\right)\left(1-\Psi\left(\gamma_{s, r}^{\tau}\right)\right)$ by knowing that $P_{r}^{h}\left(\left|y_{r, d}^{\tau}\right|>\zeta \mid \tilde{P}_{2}^{\tau}=P_{2}\right)=\mathcal{M}\left(P_{2}\left|h_{r, d}^{\tau}\right|^{2}, \zeta\right)$ and the use of the result in (13). The second term in (16) can be approximated by using the concept of conditional probability and applying Bayes' rule [13], such that $P_{r}^{h}\left(\tilde{P}_{2}^{\tau-l}=P_{2}, l=k|| y_{r, d}^{\tau-l} \mid>\zeta\right) \approx$ $\frac{\mathcal{M}\left(P_{2}\left|h_{r, d}^{\tau}\right|^{2}, \zeta\right)\left(1-\Psi\left(\gamma_{s, r}^{\tau}\right)\right)}{1-\left(1-e^{-\zeta^{2} / \mathcal{N}_{0}}\right) \Psi\left(\gamma_{s, r}^{\tau}\right)}$. Next, the chance that the scenario $\Phi_{3}$ happens can be obtained through an expression that related to $P_{r}^{h}\left(\Phi_{2}\right)$ as

where

$$
P_{r}^{h}\left(\Phi_{3}\right)=P_{r}^{h}\left(\Phi_{2} \cup \Phi_{3}\right)-P_{r}^{h}\left(\Phi_{2}\right),
$$

$$
\begin{aligned}
P_{r}^{h}\left(\Phi_{2} \cup \Phi_{3}\right) \triangleq & P_{r}^{h}\left(\left|y_{r, d}^{\tau}\right|>\zeta, \tilde{P}_{2}^{\tau}=P_{2}, \tilde{P}_{2}^{\tau-l}=P_{2},|| y_{r, d}^{\tau-l} \mid>\zeta\right) \\
= & P_{r}^{h}\left(\left|y_{r, d}^{\tau}\right|>\zeta, \tilde{P}_{2}^{\tau}=P_{2}\right) \\
& \times \frac{P_{r}^{h}\left(\left|y_{r, d}^{\tau-l}\right|>\zeta, \tilde{P}_{2}^{\tau-l}=P_{2}\right)}{P_{r}^{h}\left(\left|y_{r, d}^{\tau-l}\right|>\zeta\right)}
\end{aligned}
$$

in which the second equality results from independent signals at times $\tau-1$ and $\tau$, and the second term is obtained by applying Bayes' rule. We further evaluate the denominator of the second equality by using the concept of total probability as [13]

$$
\begin{aligned}
P\left(\left|y_{r, d}^{\tau-l}\right|>\zeta\right) & =\mathcal{M}\left(P_{2}\left|h_{r, d}^{\tau-l}\right|^{2}, \zeta\right)\left(1-\Psi\left(\gamma_{s, r}^{\tau-l}\right)\right)+e^{\frac{-\zeta^{2}}{\mathcal{N}_{0}}} \Psi\left(\gamma_{s, r}^{\tau-l}\right) \\
& \triangleq \Gamma\left(P_{1}\left|h_{s, r}^{\tau-l}\right|^{2}, P_{2}\left|h_{r, d}^{\tau-l}\right|^{2}\right) .
\end{aligned}
$$

Substituting (16) and (18)-(19) into (17), we have

$$
\begin{aligned}
P_{r}^{h}\left(\Phi_{3}\right)= & \mathcal{M}^{2}\left(P_{2}\left|h_{r, d}^{\tau}\right|^{2}, \zeta\right)\left(1-\Psi\left(\gamma_{s, r}^{\tau}\right)\right)^{2}\left(\Gamma^{-1}\left(P_{1}\left|h_{s, r}^{\tau}\right|^{2}, P_{2}\left|h_{r, d}^{\tau}\right|^{2}\right)\right. \\
& \left.-\left(1-\left(1-e^{-\zeta^{2} / \mathcal{N}_{0}}\right) \Psi\left(\gamma_{s, r}^{\tau}\right)\right)^{-1}\right) .
\end{aligned}
$$

Following the same steps as used to determine $P_{r}^{h}\left(\Phi_{2} \cup \Phi_{3}\right)$, the chance that $\Phi_{4}$ occurs can be expressed as

$$
P_{r}^{h}\left(\Phi_{4}\right)=\frac{\mathcal{M}\left(P_{2}\left|h_{r, d}^{\tau}\right|^{2}, \zeta\right) e^{\left(\frac{-\zeta^{2}}{\mathcal{N}_{0}}\right)} \Psi\left(\gamma_{s, r}^{\tau}\right)\left(1-\Psi\left(\gamma_{s, r}^{\tau}\right)\right)}{\Gamma\left(P_{1}\left|h_{s, r}^{\tau}\right|^{2}, P_{2}\left|h_{r, d}^{\tau}\right|^{2}\right)}
$$

With an assumption that the channels at time $\tau$ and time $\tau-l$ are almost the same, after some manipulations, we can find that $P_{r}^{h}\left(\Phi_{5}\right)=P_{r}^{h}\left(\Phi_{4}\right)$. Lastly, the chance that the scenario $\Phi_{6}$ occurs can be determined as

$$
\begin{aligned}
P_{r}^{h}\left(\Phi_{6}\right) & =\frac{P_{r}^{h}\left(\left|y_{r, d}^{\tau}\right|>\zeta, \tilde{P}_{2}^{\tau}=0\right) P_{r}^{h}\left(\left|y_{r, d}^{\tau-l}\right|>\zeta, \tilde{P}_{2}^{\tau-l}=0\right)}{P_{r}^{h}\left(\left|y_{r, d}^{\tau-l}\right|>\zeta\right)} \\
& =e^{\frac{-2 \zeta^{2}}{\mathcal{N}_{0}}} \Psi\left(\gamma_{s, r}^{\tau}\right)\left(\Gamma^{-1}\left(P_{1}\left|h_{s, r}^{\tau}\right|^{2}, P_{2}\left|h_{r, d}^{\tau}\right|^{2}\right)\right) .
\end{aligned}
$$

\section{Average BER analysis}

We first find the conditional BER given that each $\Phi_{i}$ happens, then derive the average BER.

1) Conditional BER of each scenario: When $\Phi_{1}$ occurs, the destination estimates the transmitted information based on only the received signals from the source. Let $\gamma_{i}$ represents the instantaneous SNR given that $\Phi_{i}$ occurs, the conditional BER for a given $\Phi_{1}$ is [11]

$$
\left.P_{B E R}^{h}\right|_{\Phi_{1}}=\Omega_{1}\left(\gamma_{1}\right) \triangleq \frac{1}{4 \pi} \int_{-\pi}^{\pi} f_{1}(\theta) \exp \left[-\alpha(\theta) \gamma_{1}\right] d \theta
$$

where $\gamma_{1}=P_{1}\left|h_{s, d}^{\tau}\right|^{2} / \mathcal{N}_{0}, f_{1}(\theta)=\frac{1-\beta^{2}}{1+2 \beta \sin \theta+\beta^{2}}$, and $\alpha(\theta)=$ $\frac{b^{2}}{2 \log _{2} M}\left(1+2 \beta \sin \theta+\beta^{2}\right)$, in which $M$ is the modulation size. The parameter $\beta=a / b$ is a constant whose value depends on $M$. For example, $a=10^{-3}$ and $b=\sqrt{2}$ for DBPSK modulation, and $a=\sqrt{2-\sqrt{2}}$ and $b=\sqrt{2+\sqrt{2}}$ for DQPSK modulation.

The conditional BERs for given $\Phi_{2}$ to $\Phi_{6}$ depend on the combining weights $a_{1}$ and $a_{2}$. Under the scenario $\Phi_{2}$, the received signals from the source and the relay are $y_{s, d}^{\tau}=v_{m} y_{s, d}^{\tau-1}+\tilde{w}_{s, d}^{\tau}$, and $y_{r, d}^{\tau}=v_{m} y_{r, d}^{\tau-l}+\tilde{w}_{r, d}^{\tau}$, respectively. Since the additive noise terms $\tilde{w}_{s, d}^{\tau}$ and $\tilde{w}_{r, d}^{\tau}$ are zero-mean Gaussian distributed with the same variances of $2 \mathcal{N}_{0}$. Therefore, the SNR of the combiner output under the scenario $\Phi_{2}$ is maximized by choosing the combining weights $a_{1}=a_{2}=1 / 2 \mathcal{N}_{0}$. Hence, $\left.P_{B E R}^{h}\right|_{\Phi_{2}}$ can be obtained from the conditional BER formulation for DMPSK signals with two-channel reception as [11]

$$
\left.P_{B E R}^{h}\right|_{\Phi_{2}}=\Omega_{2}\left(\gamma_{2}\right) \triangleq \frac{1}{16 \pi} \int_{-\pi}^{\pi} f_{2}(\theta) \exp \left[-\alpha(\theta) \gamma_{2}\right] d \theta,
$$

where $f_{2}(\theta)=\frac{b^{2}\left(1-\beta^{2}\right)\left[3+\cos (2 \theta)-\left(\beta+\frac{1}{\beta}\right) \sin (\theta)\right]}{2 \alpha(\theta)}$, and $\beta$ and $\alpha(\theta)$ are as specified in (23), and the instantaneous SNR is $\gamma_{2}=$ $P_{1}\left|h_{s, d}^{\tau}\right|^{2} / \mathcal{N}_{0}+P_{2}\left|h_{r, d}^{\tau}\right|^{2} / \mathcal{N}_{0}$.

In case of $\Phi_{3}$ to $\Phi_{6}$, the destination uses the two-channel differential detection which are not guaranteed to be optimum since either $y_{r, d}^{\tau}$ or $y_{r, d}^{\tau-l}$ may contains only noise. Up to now, the conditional BER formulation for DMPSK with arbitrary-weighted combining has not been available in the literature. For analytical tractability, we resort to an approximate BER, in which the signal from the relay is considered as noise when $\Phi_{3}$ to $\Phi_{6}$ occur. As we will show in the succeeding section, the BER obtained from this approximation is very close to the simulated performance. We can write the approximate conditional BER for the scenarios $\Phi_{\nu}, \nu=3, \ldots, 6$, as $\left.P_{B E R}^{h}\right|_{\Phi_{\nu}} \approx \Omega_{2}\left(\gamma_{\nu}\right)$, where

$$
\gamma_{\nu}=P_{1}\left|h_{s, d}^{\tau}\right|^{2} /\left(\mathcal{N}_{0}+\mathcal{N}_{\nu} /\left(P_{1}\left|h_{s, d}^{\tau}\right|^{2} / \mathcal{N}_{0}\right)\right)
$$

in which $\mathcal{N}_{\nu}$ represents the noise power that comes from the relay link given that the scenario $\Phi_{\nu}$ occurs. Depending on the received signals $y_{r, d}^{\tau}$ and $y_{r, d}^{\tau-l}, \mathcal{N}_{\nu}$ can be specified as: $\mathcal{N}_{3}=$ $\left(P_{2}\left|h_{r, d}^{\tau}\right|^{2}+\mathcal{N}_{0}\right)^{2} / \mathcal{N}_{0}, \mathcal{N}_{4}=\mathcal{N}_{5}=P_{2}\left|h_{r, d}^{\tau}\right|^{2}+\mathcal{N}_{0}$, and $\mathcal{N}_{6}=\mathcal{N}_{0}$.

2) Average BER: The average BER, $P_{B E R}$, is obtained by the summation of the average BERs of all six scenarios as

$$
P_{B E R}=P_{B E R}^{(1)}+P_{B E R}^{(2)}+P_{B E R}^{(3)}+2 P_{B E R}^{(4)}+P_{B E R}^{(6)},
$$

in which $P_{B E R}^{(i)} \triangleq \mathrm{E}\left[\left.P_{B E R}^{h}\right|_{\Phi_{i}} P_{r}^{h}\left(\Phi_{i}\right)\right]$ represents an average BER for each scenario which can be obtained by averaging the results in Section III-B and III-C.1 over all fading channels. We summarize here the final results due to space limitation. Assuming that the fading channels at different transmit-receive links are independent, the average BER for each scenario $\Phi_{i}$ is as follows

$$
\begin{aligned}
P_{B E R}^{(1)}= & F_{1}\left(1+\alpha(\theta) P_{1} \sigma_{s, d}^{2} / \mathcal{N}_{0}\right)\left[\left(1-e^{\frac{-\zeta^{2}}{\mathcal{N}_{0}}}\right) G\left(1+g(\theta) P_{1} \sigma_{s, r}^{2} / \mathcal{N}_{0}\right)\right. \\
& \left.+\left(1-\int_{0}^{\infty} \frac{\mathcal{M}\left(P_{2} q, \zeta\right)}{\sigma_{r, d}^{2}} e^{\frac{-q}{\sigma_{r, d}^{2}}} d q\right)\left(1-G\left(1+\frac{g(\theta) P_{1} \sigma_{s, r}^{2}}{\mathcal{N}_{0}}\right)\right)\right]
\end{aligned}
$$

in which $F_{1}(c(\theta))=\frac{1}{4 \pi} \int_{-\pi}^{\pi} f_{1}(\theta) / c(\theta) d \theta$, and $G(c(\theta))=$ $\frac{1}{\pi} \int_{0}^{(M-1) \pi / M} c(\theta)^{-1} d \theta$, in which $c(\theta)$ is a function of $\theta$. 


$$
\begin{aligned}
P_{B E R}^{(2)} \approx & \frac{1}{\sigma_{r, d}^{2}} \int_{0}^{\infty} s_{2}(q) \mathcal{M}^{2}\left(P_{2} q, \zeta\right) e^{-\frac{q}{\sigma_{r, d}^{2}}} d q \\
& \cdot \frac{1}{\sigma_{s, r}^{2}} \int_{0}^{\infty} \frac{\left(1-\Psi\left(P_{1} u / \mathcal{N}_{0}\right)\right)^{2}}{1-\left(1-e^{-\zeta^{2} / \mathcal{N}_{0}}\right) \Psi\left(P_{1} u / \mathcal{N}_{0}\right)} e^{-\frac{u}{\sigma_{s, r}^{2}}} d u,
\end{aligned}
$$

in which $s_{2}(q)=\frac{1}{16 \pi} \int_{-\pi}^{\pi} f_{2}(\theta)\left(1+\frac{\alpha(\theta) P_{1} \sigma_{s, d}^{2}}{\mathcal{N}_{0}}\right)^{-1} e^{\left(-\alpha(\theta) \frac{P_{2} q}{\mathcal{N}_{0}}\right)} d \theta$.

$$
P_{B E R}^{(3)} \approx \frac{1}{\sigma_{s, r}^{2}} \int_{0}^{\infty}\left[\frac{1}{16 \pi} \int_{-\pi}^{\pi} f_{2}(\theta) s_{3}(u, \theta) d \theta\right] e^{-u / \sigma_{s, r}^{2}} d u,
$$

in which

$$
\begin{aligned}
& s_{3}(u, \theta) \\
& =\frac{1}{\sigma_{s, d}^{2} \sigma_{r, d}^{2}} \int_{0}^{\infty} \int_{0}^{\infty} \exp \left(-\frac{\alpha(\theta) P_{1} z}{\mathcal{N}_{0}+\frac{\left(P_{2} q+\mathcal{N}_{0}\right)^{2}}{P_{1} z}}-\frac{z}{\sigma_{s, d}^{2}}-\frac{q}{\sigma_{r, d}^{2}}\right) \\
& \times \mathcal{M}^{2}\left(P_{2} q, \zeta\right)\left(1-\Psi\left(P_{1} u / \mathcal{N}_{0}\right)\right)^{2} \\
& \times\left(\frac{1}{\Gamma\left(P_{1} u, P_{2} q\right)}-\frac{1}{1-\left(1-e^{-\zeta^{2} / \mathcal{N}_{0}}\right) \Psi\left(P_{1} u / \mathcal{N}_{0}\right)}\right) d q d z . \\
& P_{B E R}^{(4)}=P_{B E R}^{(5)} \approx \frac{1}{\sigma_{s, r}^{2}} \int_{0}^{\infty}\left[\frac{1}{16 \pi} \int_{-\pi}^{\pi} f_{2}(\theta) s_{4}(u, \theta) d \theta\right] e^{-u / \sigma_{s, r}^{2}} d u, \\
& =\frac{1}{\sigma_{s, d}^{2} \sigma_{r, d}^{2}} \int_{0}^{\infty} \int_{0}^{\infty} \exp \left(-\frac{\alpha(\theta) P_{1} z}{\mathcal{N}_{0}+\frac{P_{2} q+\mathcal{N}_{0}}{P_{1} z / \mathcal{N}_{0}}}-\frac{\zeta^{2}}{\mathcal{N}_{0}}-\frac{z}{\sigma_{s, d}^{2}}-\frac{q}{\sigma_{r, d}^{2}}\right) \\
& \times \mathcal{M}\left(P_{2} q, \zeta\right) \frac{\Psi\left(P_{1} u / \mathcal{N}_{0}\right)\left(1-\Psi\left(P_{1} u / \mathcal{N}_{0}\right)\right)}{\Gamma\left(P_{1} u, P_{2} q\right)} d q d z . \\
& P_{B E R}^{(6)} \approx \frac{e^{-2 \zeta^{2} / \mathcal{N}_{0}}}{\sigma_{s, r}^{2} \sigma_{r, d}^{2}} \int_{0}^{\infty} \int_{0}^{\infty} \frac{\Psi^{2}\left(P_{1} u / \mathcal{N}_{0}\right)}{\Gamma\left(P_{1} u, P_{2} q\right)} e^{-\frac{u}{\sigma_{s, r}^{2}}-\frac{q}{\sigma_{r, d}^{2}}} d q d u \\
& \times \frac{1}{16 \pi \sigma_{s, d}^{2}} \int_{-\pi}^{\pi} f_{2}(\theta) \int_{0}^{\infty} e^{\left(-\frac{\alpha(\theta) P_{1} z}{\mathcal{N}_{0}+\mathcal{N}_{0}^{2} / P_{1} z}-\frac{z}{\sigma_{s, d}^{2}}\right)} d z d \theta .
\end{aligned}
$$

\section{BER UPPER BOUND AND BER LOWER BOUND}

To obtain a BER upper bound, we first note that the conditional BER for each case, $\left.P_{B E R}^{h}\right|_{i}$, is at most $1 / 2$. In addition, if the threshold is properly designed, the chances that $\Phi_{3}$ to $\Phi_{6}$ happen are small compared to the chances that $\Phi_{1}$ and $\Phi_{2}$ happen. By bounding the conditional BER $\left.P_{B E R}^{h}\right|_{\Phi_{3}}, P_{B E R}^{h}\left|\Phi_{4}, P_{B E R}^{h}\right|_{\Phi_{5}}$, and $\left.P_{B E R}^{h}\right|_{\Phi_{6}}$ by $1 / 2$, the BER upper bound is given by

$$
P_{B E R} \leq P_{B E R}^{(1)}+P_{B E R}^{(2)}+\frac{1}{2}\left\{P_{r}^{h}\left(\Phi_{3}\right)+2 P_{r}^{h}\left(\Phi_{4}\right)+P_{r}^{h}\left(\Phi_{6}\right)\right\} .
$$

Next, we determine a BER lower bound by observing that the conditional BER under the scenario $\Phi_{3}\left(\left.P_{B E R}^{h}\right|_{\Phi_{3}}\right)$ is larger than $\left.P_{B E R}^{h}\right|_{\Phi_{2}}$. Therefore, the BER lower bound can be obtained by bounding the conditional BER $\left.P_{B E R}^{h}\right|_{\Phi_{3}}$ with $\left.P_{B E R}^{h}\right|_{\Phi_{2}}$, we have

$$
P_{B E R}^{(2)}+P_{B E R}^{(3)} \geq \mathrm{E}\left[P_{B E R}^{h} \mid \Phi_{2} P_{r}^{h}\left(\Phi_{2} \cup \Phi_{3}\right)\right],
$$

where $P_{r}^{h}\left(\Phi_{2} \cup \Phi_{3}\right)$ is evaluated in (18). By averaging (28) over all channel realizations, we have

$$
\begin{aligned}
P_{B E R}^{(2)}+P_{B E R}^{(3)} & \geq \frac{1}{16 \pi \sigma_{s, r}^{2}} \int_{0}^{\infty}\left[\int_{-\pi}^{\pi} \frac{f_{2}(\theta) s(u, \theta)}{1+\alpha(\theta) \frac{P_{1} \sigma_{s, d}^{2}}{\mathcal{N}_{0}}} d \theta\right] e^{-\frac{u}{\sigma_{s, r}^{2}}} d u . \\
& \triangleq \mathrm{LB}\left\{P_{B E R}^{(2)}+P_{B E R}^{(3)}\right\}
\end{aligned}
$$

$s(u, \theta)=\int_{0}^{\infty} \frac{\mathcal{M}^{2}\left(P_{2} q, \zeta\right)\left(1-\Psi\left(\frac{P_{1} u}{\mathcal{N}_{0}}\right)\right)^{2}}{\sigma_{r, d}^{2} \Gamma\left(P_{1} u, P_{2} q\right)} e^{-\alpha(\theta) \frac{P_{2} q}{\mathcal{N}_{0}}-\frac{q}{\sigma_{r, d}^{2}}} d q$.

Since the exact BER formulations under the scenarios 4,5 , and 6 are currently unavailable, and the chances that these three

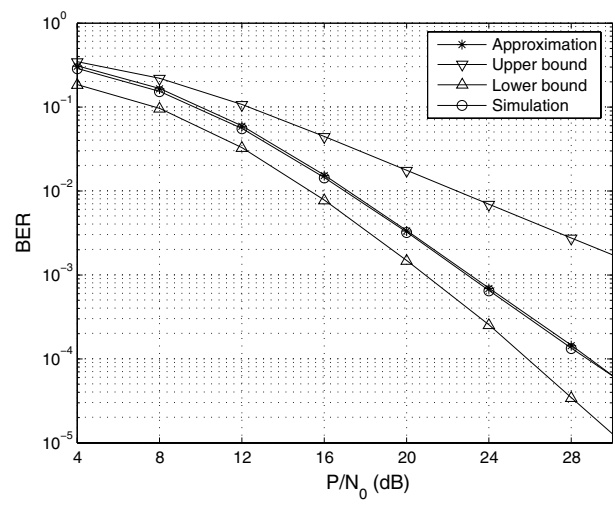

Fig. 3: DQPSK: $P_{1}=P_{2}=0.5 P, \zeta=1, \sigma_{s, d}^{2}=\sigma_{s, r}^{2}=\sigma_{r, d}^{2}=1$.

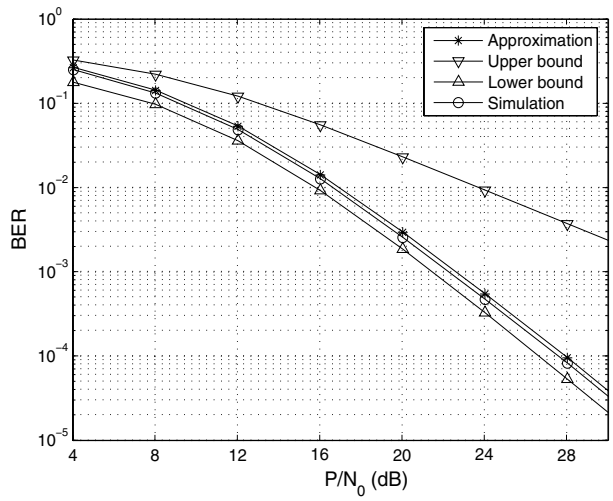

Fig. 4: DQPSK: $P_{1}=0.8 P, P_{2}=0.2 P, \zeta=1, \sigma_{s, d}^{2}=\sigma_{s, r}^{2}=\sigma_{r, d}^{2}=1$. scenarios happen are small at high SNR, we further bound $P_{B E R}^{(4)}$, $P_{B E R}^{(5)}$, and $P_{B E R}^{(6)}$ by 0 . Then, we obtain the lower bound

$$
P_{B E R} \geq P_{B E R}^{(1)}+\operatorname{LB}\left\{P_{B E R}^{(2)}+P_{B E R}^{(3)}\right\} .
$$

Figures 3-4 compare the BER approximation (26), the BER upper bound (27), and the BER lower bound (30) with the simulated performance in case of DQPSK modulation. We consider a case that $\sigma_{s, d}^{2}=\sigma_{s, r}^{2}=\sigma_{r, d}^{2}=1$ and $\zeta=1$. From both Figures, we observe that the approximate BER closely matches with the simulated BER, and both the approximate BER and the simulated BER lie between the BER upper bound and the BER lower bound. Moreover, by choosing proper power allocation and threshold, not only the BER performance improves, but also the lower bound is closer to the simulated performance. Specifically, changing the power allocation from $P_{1} / P=0.5$ to $P_{1} / P=0.8$ where $P=P_{1}+P_{2}$ is the total transmitted power, the BER performance is improved by $1 d B$ at a BER of $10^{-4}$, while the performance gap between the simulated BER and the BER lower bound is reduced by $2 d B$. The reason is that when the threshold $\zeta$ is appropriately chosen, the scenarios $\Phi_{3}$ to $\Phi_{6}$ occur with much smaller probabilities than the scenarios $\Phi_{1}$ and $\Phi_{2}$. Even though the conditional BER under each of scenarios $\Phi_{3}$ to $\Phi_{6}$ is larger than that under the scenarios $\Phi_{1}$ or $\Phi_{2}$, the average BER $P_{B E R}^{(\nu)}$, $\nu=3, \ldots, 6$, are smaller.

\section{Optimum Threshold AND Power Allocation}

We determine in this section joint optimum decision threshold and optimum power allocation based on the tight BER approximation in (26). To simplify the notation, let us denote $r=P_{1} / P$ as the power ratio of the transmitted power at the source $\left(P_{1}\right)$ over the total power $(P)$. For a fixed $P$, we jointly optimize 


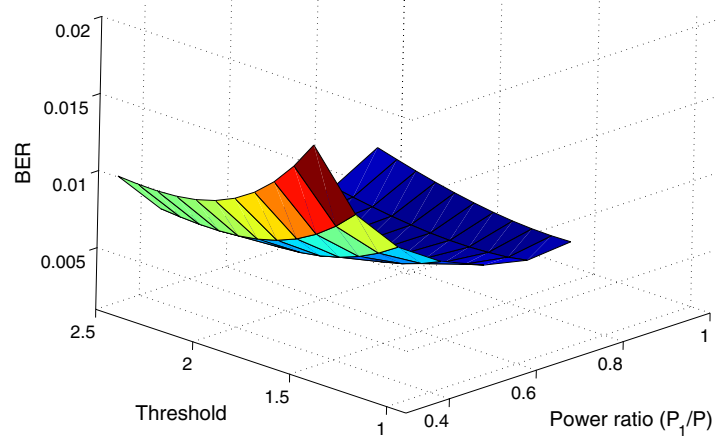

(a)

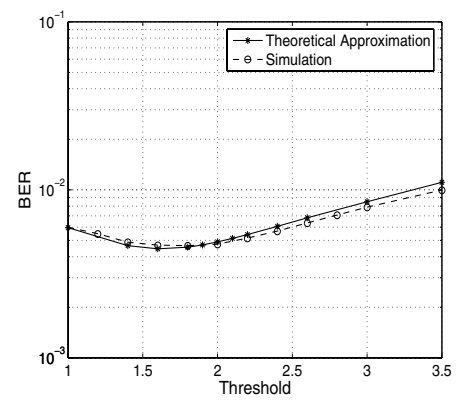

(b)

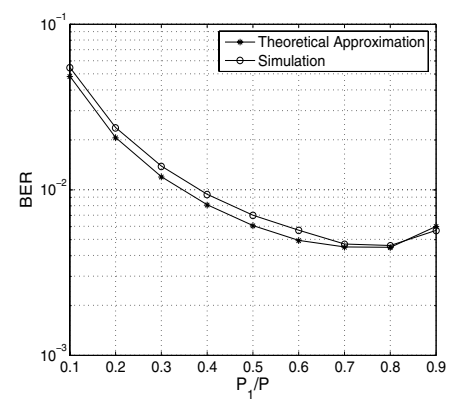

(c)
Fig. 5: DQPSK: $\sigma_{s, d}^{2}=\sigma_{s, r}^{2}=1, \sigma_{r, d}^{2}=10$ : (a) Joint optimum threshold and power allocation, (b) varying threshold with fixed power allocation at $P_{1}=0.8 P, P_{2}=0.2 P$, and (c) varying power allocation with fixed threshold at $\zeta=1.7$.

the threshold $\zeta$ and the power ratio $r$ such that the tight BER approximation in (26) is minimized:

$$
(\hat{\zeta}, \hat{r})=\arg \min _{\zeta, r} P_{B E R}(\zeta, r),
$$

where $P_{B E R}(\zeta, r)$ represents the BER approximation as specified in (26) with $P_{1}=r P$ and $P_{2}=(1-r) P$.

Figures 5(a)-5(c) show the BER performance of the proposed scheme with DQPSK signals under different power allocations and thresholds. We consider a case that $\sigma_{s, d}^{2}=1, \sigma_{s, r}^{2}=1$, $\sigma_{r, d}^{2}=10$. The BER approximation is plotted in Figure 5(a) and its cross sections are shown in Figure 5(b) and 5(c) together with the simulated BER curves. By numerically evaluation of the optimization problem in (31), Figure 5(a) shows that the joint optimum power allocation and decision threshold are $r=0.8$ and $\zeta=1.7$. Figure $5(\mathrm{~b})$ shows cross sectional curves of the approximate BER and the simulated BER performance under power allocation $r=0.8$ and different thresholds. We can see that the approximate BER closely matches to the simulated BER for all thresholds. According to both the simulated BER and the approximate BER, the optimum threshold for this case is about 1.7. We show in Figure 5(c) a comparison of the approximate BER and the simulated BER with decision threshold $\zeta=1.7$ under different power allocation. Clearly, the approximate BER follows the same trend as the simulated BER, and the optimum power allocation is about $r=0.8$.

\section{Simulation Results}

We perform computer simulations of the threshold-based differential DF scheme. The channel coefficients are modeled according to the Jakes' model [14] with the Doppler frequency $f_{D}=75 \mathrm{~Hz}$ and normalized fading parameter $f_{D} T_{s}=0.0025$ where $T_{s}$ is the sampling period. The noise variance is $\mathcal{N}_{0}=1$. We plot the BER performance curves as functions of $P / \mathcal{N}_{0}$, where $P$ is the total transmitted power. For demonstration purpose, the DQPSK modulation is employed, and channel variances are $\sigma_{s, d}^{2}=1$, $\sigma_{s, r}^{2}=1$, and $\sigma_{r, d}^{2}=10$ in all simulations.

Figure 6 compares the performance of the proposed thresholdbased differential DF scheme to that of the differential DF scheme without threshold and that of the differential DF scheme in which the relay always forwards the decoded symbols to the destination. The power allocation is $P_{1}=0.5 P, P_{2}=0.5 P$. The proposed differential DF scheme outperforms the other two schemes. The reason is that a decoding error at the relay tends to result in an error at the destination. Hence, forwarding all decoded symbols results in worse performance than that of the propose scheme. Moreover, the performance can be further improved by adding a threshold at the destination: the proposed scheme yields about $4 d B$ gain at a BER of $10^{-3}$ compared to the scheme without threshold. Furthermore, the proposed scheme shows $5 d B$ performance gap in comparison to its coherent counterpart, but the differential scheme without a decision threshold losses about $9 d B$ in comparison to its coherent counterpart at a BER of $10^{-3}$.

We show in Figure 7 the BER performance in case of power allocation $P_{1}=0.8 P$ and $P_{2}=0.2 P$. Obviously, different thresholds result in different BER performances. The threshold of $\zeta=1.7$ provides the best performance in this scenario. If the threshold is too small, e.g. $\zeta=1$, not only the BER performance degrades but also the diversity order is less than two. This is because when the threshold is small, the destination tends to combine the signals from both the relay and the destination. As a result, the incorrect decoding at the relay leads to significant performance degradation.

In Figure 8, we study the effect of power allocation on the BER performance with a fixed threshold at $\zeta=1$. Based on the numerical evaluation of (31), the optimum power ratio for this scenario is $r=0.9$. We can see that the simulation results in Figure 8 agree with the obtained numerical results. Moreover, Figure 8 illustrates that the power ratio of $r=0.9$ results in optimum performance over the entire SNR range. We observe that the proposed scheme with optimum power allocation achieves about $5 d B$ improvement over that with equal power allocation at a BER of $10^{-4}$.

Figure 9 compares the performances with different power allocations and decision thresholds. We can see that the joint optimum power allocation and optimum threshold yields the best performance over the entire SNR range. When the quality of the relay-destination link is very good, e.g. $\sigma_{r, d}^{2}=10$, at high SNR, using the optimum threshold is more important than using the optimum power allocation. Specifically, by properly choosing the threshold, the proposed differential DF scheme achieves almost the same performance for any power allocation at high SNR. In case of equal power allocation, using the optimum threshold leads to more than $5 \mathrm{~dB}$ gain over the scheme without threshold at a BER of $10^{-4}$. With the optimum threshold, using the optimum power allocation improves only $0.5 d B$ at a BER of $10^{-4}$ compared to the equal power allocation scheme. 


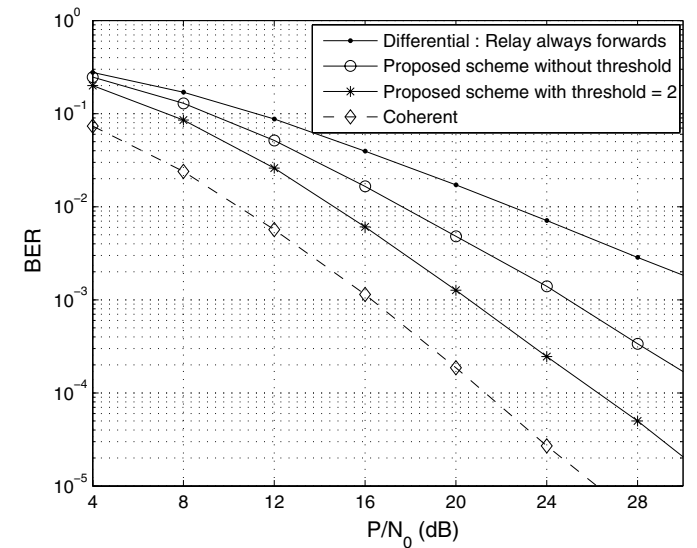

Fig. 6: DQPSK with different cooperation schemes, $P_{1}=P_{2}=0.5 P$, $\sigma_{s, d}^{2}=1, \sigma_{s, r}^{2}=1$, and $\sigma_{r, d}^{2}=10$.

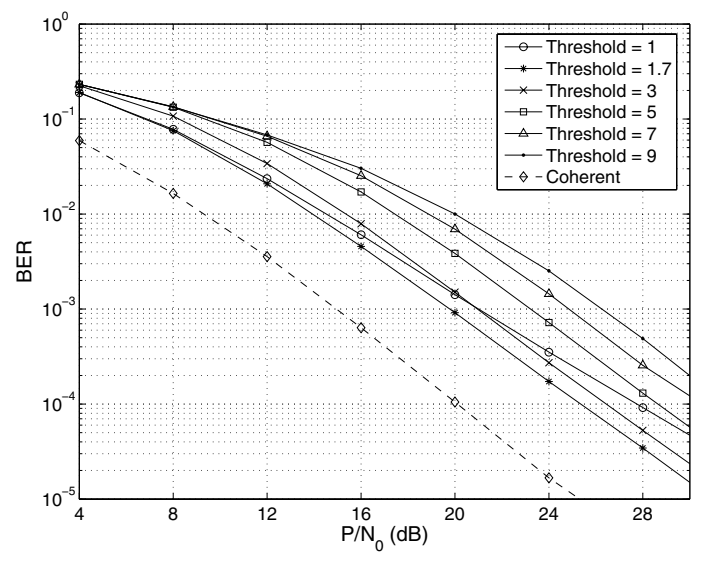

Fig. 7: DQPSK: Different thresholds with fixed power allocation, $P_{1}=$ $0.8 P, P_{2}=0.2 P, \sigma_{s, d}^{2}=\sigma_{s, r}^{2}=1, \sigma_{r, d}^{2}=10$.

\section{CONCLUSIONS}

We propose a threshold-based differential decode-and-forward scheme for a two-user cooperation system. By allowing the relay forwards only the correctly decoded symbols and introducing a decision threshold at the destination, the proposed scheme efficiently combines the signals from the direct and the relay links. An approximate BER expression is derived for differential M-ary phase shift keying (DMPSK) modulation, and the BER lower bound and BER upper bound are formulated. The approximate BER is very close to the simulated BER curve, and it lies between the obtained BER lower bound and BER upper bound. Base on the tight BER approximation, we determine the optimum decision threshold and power allocation numerically. When the quality of the relay-destination link is much larger than the other links, i.e., $\sigma_{s, d}^{2}=\sigma_{s, r}^{2}=1$ and $\sigma_{r, d}^{2}=10$, then the decision threshold is more important than the power allocation at high SNR. For instance, in case of DQPSK signals with equal power allocation, using the optimum threshold results in more than $5 \mathrm{~dB}$ gain over the scheme without threshold at a BER of $10^{-4}$. By further using the optimum power allocation, the performance improvement is about $0.5 d B$ at the same BER.

\section{REFERENCES}

[1] M. K. Simon, S. M. Hinedi, and W. C. Lindsey, Digital Communication Techniques-Signal Design and Detection. Englewood Cliffs, NJ: Prentice Hall, 1994.

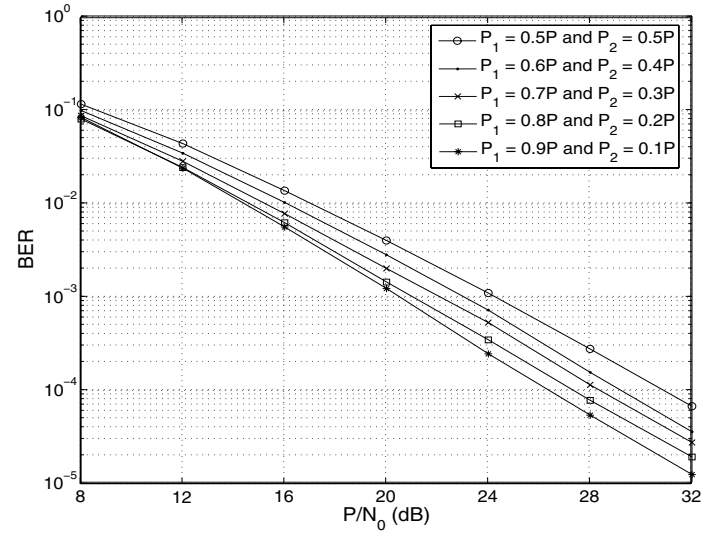

Fig. 8: DQPSK: Different power allocations with fixed threshold, $\zeta=$ $1, \sigma_{s, d}^{2}=\sigma_{s, r}^{2}=1, \sigma_{r, d}^{2}=10$.

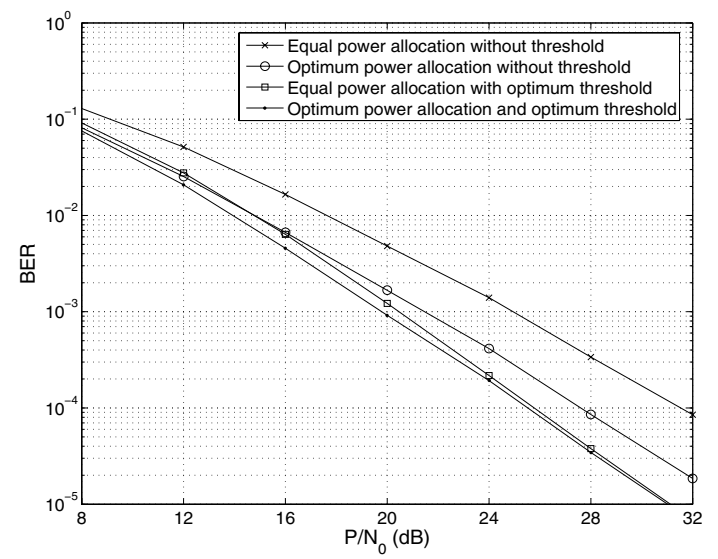

Fig. 9: DQPSK: Different power allocations and different thresholds, $\sigma_{s, d}^{2}=\sigma_{s, r}^{2}=1, \sigma_{r, d}^{2}=10$.

[2] B. M. Hochwald and W. Sweldens, "Differential unitary space-time modulation," IEEE Trans. Commun., vol. 48, pp. 2041-2052, Dec. 2000.

[3] J. N. Laneman, G. W. Wornell, and D. N. C. Tse, "An efficient protocol for realizing cooperative diversity in wireless networks," in Proc. IEEE Intl. Symp. Inform. Theory, (ISIT), Washington, DC, Jun. 2001.

[4] J. N. Laneman, D. N. C. Tse, and G. W. Wornell, "Cooperative diversity in wireless networks: efficient protocols and outage behavior," IEEE Trans. Inform. Theory, vol. 50, no. 12, pp. 3062-3080, Dec. 2004.

[5] M. Janani, A Hedayat, T. E. Hunter and A. Nosratinia, "Coded cooperation in wireless communications: space-time transmission and iterative decoding," IEEE Trans. Signal Processing, vol. 52, pp. 362-370, Feb. 2004.

[6] M. O. Hasna and M.-S. Alouini, "Performance analysis of two-hop relayed transmissions over Rayleigh fading channels," in Proc. IEEE Vehicular Tech. Conf. (VTC), vol. 4, pp. 1992-1996, Sept. 2003.

[7] W. Su, A. K. Sadek, and K. J. R. Liu, "SER performance analysis and optimum power allocation for decode-and-forward cooperation protocol in wireless networks" in Proc. IEEE WCNC, vol. 2, pp. 984-989, Mar. 2005.

[8] A. K. Sadek, W. Su, and K. J. R. Liu, "A class of cooperative communication protocols for multi-node wireless networks," in Proc. IEEE SPAWC, New York city, NY, pp. 560-564, Jun. 2005.

[9] D. Chen and J. N. Laneman, "Noncoherent demodulation for cooperative diversity in wireless systems," IEEE Global Comm. Conf. (GLOBECOM), Dallas, TX, pp. 31-35, Nov. 2004.

[10] P. Tarasak, H. Minn, and V. K. Bhargava, "Differential modulation for twouser cooperative diversity systems," IEEE Global Comm. Conf. (GLOBE COM), Dallas, TX, Nov. 2004.

[11] M. K. Simon and M.-S. Alouini, "A unified approach to the probability of error for noncoherent and differentially coherent modulations over generalized fading channels," IEEE Trans. Commun., vol. 46, no. 12, pp. 1625-1638, Dec. 1998.

[12] R. F. Pawula, "Generic error probabilities," IEEE Trans. Commun., vol. 47, no. 5, pp. 697-702, May 1999.

[13] H. Stark and J. W. Woods, Probability and Random Processes with Applications to Signal Processing. Prentice Hall, 2001.

[14] G. Stuber, Principle of Mobile Communications. Kluwer Academic Publishers, $2^{\text {nd }}$ ed., 2001. 